\title{
Atomic-Scale Characterization Reveals Core-Shell Structure of Enamel Crystallites
}

\author{
Paul JM Smeets ${ }^{1}$, Karen DeRocher ${ }^{1}$, Michael J Zachman ${ }^{2}$, Berit H Goodge ${ }^{2}$, Lena F Kourkoutis ${ }^{2}$ and \\ Derk Joester $^{1 *}$ \\ 1. Northwestern University, Department of Materials Science and Engineering, Chicago, IL, USA. \\ 2. Cornell University, School of Applied and Engineering Physics, Ithaca, NY, USA. \\ * Corresponding author: d-joester@northwestern.edu
}

Human enamel is the hardest tissue in our body (hardness $~ 2-6 \mathrm{GPa}$ ), due to its high mineral content (96 $\mathrm{wt} \%$ ) and its complex 3D hierarchical structure across different length scales (Fig. 1). The characteristic microstructural unit of enamel - the enamel rod (Fig. 1A) - is comprised of thousands of lath-like crystallites (Fig. 1B) nominally composed of hydroxylapatite $\left(\mathrm{OHAp}\right.$; $\mathrm{Ca}_{5}\left(\mathrm{PO}_{4}\right)_{3}(\mathrm{OH})(\mathrm{Fig}$. $1 \mathrm{C} \&$ Fig. $1 \mathrm{C}$, inset)). However, enamel is known to contain small quantities of other ions, such as $\mathrm{Mg}^{2+}(0.2-0.6$ $\mathrm{wt} \%), \mathrm{Na}^{+}, \mathrm{CO}_{3}{ }^{2-}$, and $\mathrm{F}^{-}$, which can substitute in the OHAp lattice and are known to increase $\left(\mathrm{Mg}^{2+}\right.$, $\left.\mathrm{Na}^{+}, \mathrm{CO}_{3}{ }^{2-}\right)$ or decrease $\left(\mathrm{F}^{-}\right)$its solubility [1]. These minor components play a key role in how enamel is affected by exposure to acid in the oral cavity, which may lead to tooth decay (caries). Therefore, knowledge of the ion distribution on the length scale of individual crystallites is vital in understanding processes of caries formation and progression, which eventually may lead to novel treatment methods.

Studying enamel using conventional electron microscopy at such length scales is intricate due to its severe sensitivity to electron beam damage. We therefore performed aberration-corrected scanning TEM imaging of focused ion beam prepared human enamel sections at cryogenic temperature, to minimize inelastic scattering damage and reduce carbon contamination [2]. Moreover, we used probe conditions (4.0-8.0 pA; dwell time 1-2 $\mu \mathrm{s})$ below the electron dose threshold for OHAp mass loss $\left(\sim 900 \mathrm{e}^{-} / \AA^{2}\right)$. Several images were collected, registered by cross-correlation, and averaged to minimize noise (Fig. 1D) [3]. A careful evaluation of crystallites using medium angle annular dark field (MAADF) imaging revealed that crystallites are clearly separated by thin regions containing lower atomic number $(Z)$ contrast (Fig. 2A). In addition, although crystallites appear as single crystals (Fig. 2A,B, inset), they contain a core-shell structure, with a shell appearing brighter compared to a darker (lower Z-contrast) core (Fig. 2A). Thus, both the intergranular regions and the core should contain lower $Z$ elements.

We investigated this hypothesis by performing electron energy loss spectroscopy (EELS) using a K2 direct electron detector for high speed acquisition of spectra with low background noise. Core-loss spectra $(\mathrm{Ca}, \mathrm{P}, \mathrm{O}$ and $\mathrm{C}$ ) from enamel crystallites were obtained, consistent with the major constituents of human tooth enamel (Fig. 2C). In addition, we obtained low-loss $\mathrm{Mg} L_{2,3}$ edges ( $\sim 2 \mathrm{eV}$ and $\sim 75 \mathrm{eV}$; Fig. 2D), representing $\mathrm{Mg}$ ions that are in different chemical or structural environments. Multivariate curve resolution (MCR) was used to decompose $\mathrm{Mg} L_{2,3}$ spectra into component profiles ( $\mathrm{Mg} 1$ and $\mathrm{Mg}$ 2, Fig. 2D) and determine intensity maps (Fig. 2F). Inspection of maps revealed that $\mathrm{Mg} 1$ is evenly distributed within the crystallite, while $\mathrm{Mg} 2$ localizes to areas surrounding the periphery of the crystallites. Additionally, Mg 2 appears to be distributed preferentially within the core region. Together, this indicates that the $\mathrm{Mg}$ components serve as a probe for distinguishing 1) intergranular regions from the enamel crystallites, and 2) the crystallite core from its shell, both confirming the lower Z-contrast contrast observations in these regions. To accurately quantify $\mathrm{Mg}$ concentrations, atom probe tomography (APT) analysis revealed a higher $\mathrm{Mg}$ content in the core and intergranular regions $(\sim 1$ at $\%)$, compared to a lower Mg content in the shell of the crystallites ( $\sim 0.3 \mathrm{at} \%$ ) (Fig. $2 \mathrm{G})$. The result is that the 
solubility in the core and intergranular regions should be increased through substitution of $\mathrm{Mg}^{2+}$ for $\mathrm{Ca}^{2+}$ in the OHAp lattice, which can be confirmed when acid-etching enamel sections (Fig. 2H). Thus, for the first time we image a section of human enamel at atomic resolution, quantify and localize chemical impurity content within the crystallites, and discuss the implications for dental caries formation.
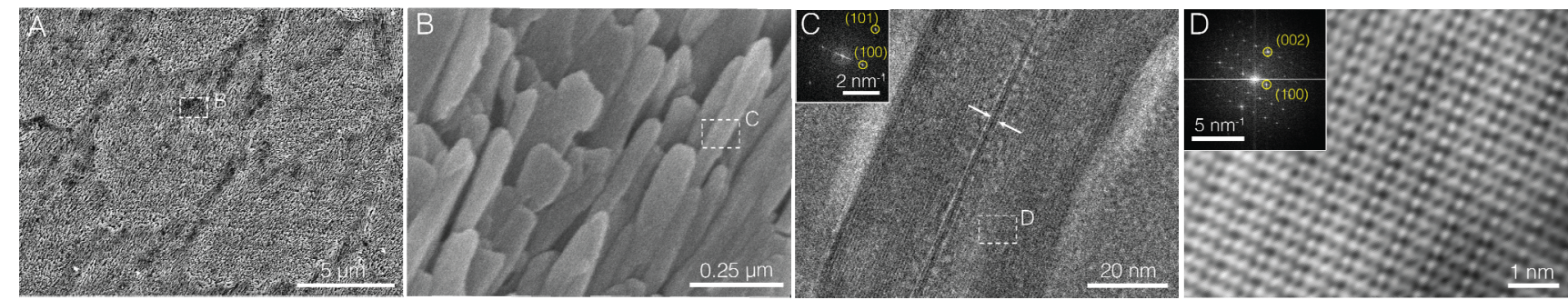

Figure 1. A) SEM image of enamel rods in etched outer enamel. B) SEM image of OHAp crystallites that constitute the enamel rod. C) HRTEM image of a crystallite close to the [010] zone axis (ZA) (inset: FFT). Note the central dark line (CDL) feature (white arrows). D) Aberration-corrected STEMMAADF lattice image of a crystallite viewed parallel to the [010] ZA (inset: FFT).
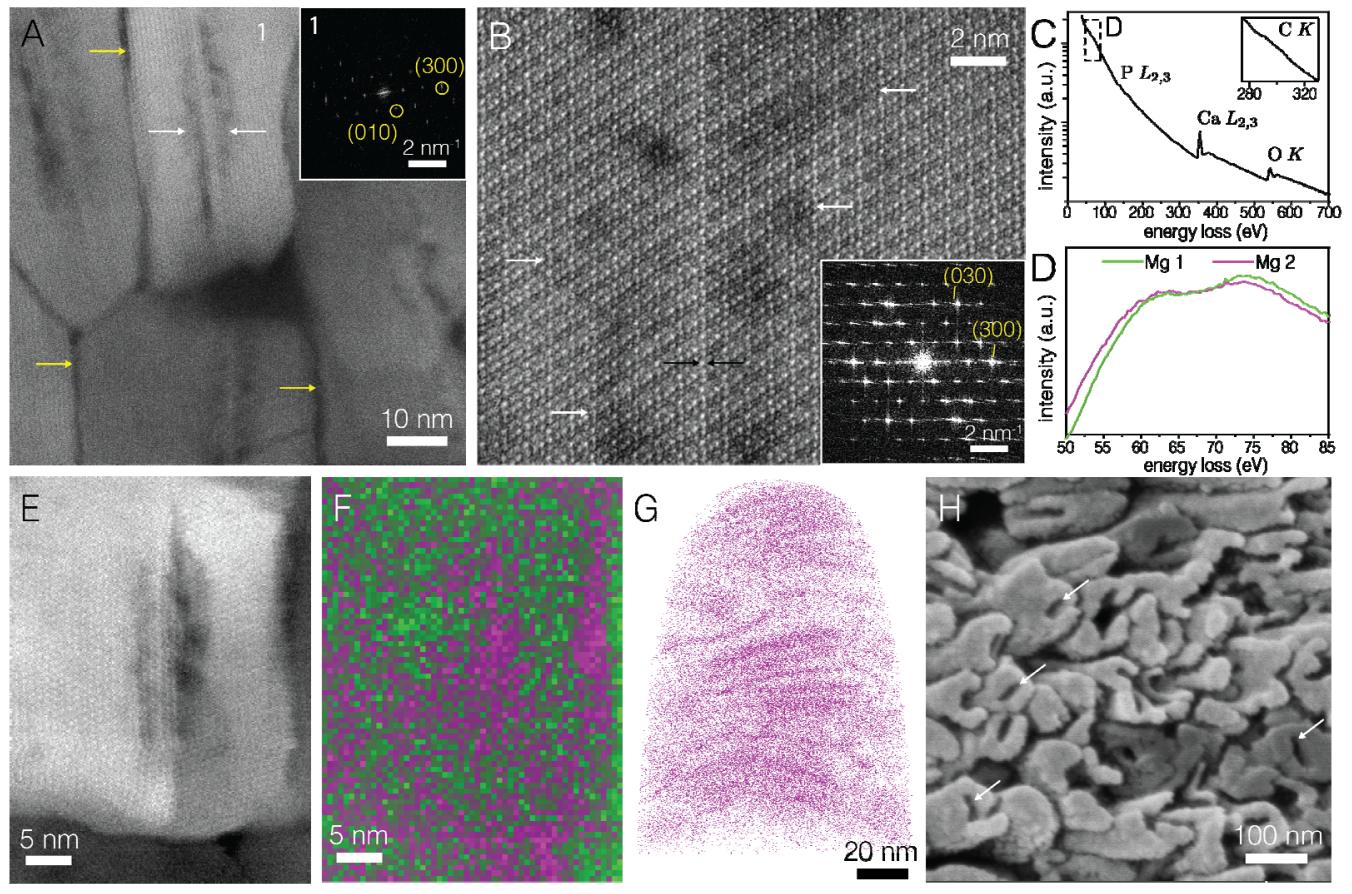

Figure 2. A) MAADF image of enamel crystallites oriented (close) to the [001] ZA. (inset: FFT from crystallite 1). Yellow arrows indicate intergranular regions, while white arrows indicate the crystallite core. B) MAADF lattice image of central part of a single crystallite oriented to the [001] ZA. The CDL (black arrows), runs parallel to the $\{100\}$ set of planes. Inset: FFT. C) Typical EEL spectrum obtained from nanoscale regions of crystallites (inset: $\mathrm{C} K$-edge). D) MCR of $\mathrm{Mg} L_{2,3}$ edges. E) MAADF image of a crystallite along the [001] direction, with corresponding F) composite map of $\mathrm{Mg} 1$ (green) and $\mathrm{Mg}$ 2 (magenta). G) APT 3D reconstruction of $\mathrm{Mg}^{2+}$ ions. H) SEM image showing edge-on crystallites of an acid-etched enamel section. White arrows point to dissolution of the core from individual crystallites.

[1] C Robinson, et al., Critical Reviews in Oral Biology \& Medicine 11 (2000), p. 15.

[2] Y. Talmon in "Electron Beam Radiation Damage to Organic and Biological Cryospecimens", ed. R.A. Steinbrecht and K. Zierold, (Springer, Berlin), p. 64.

[3] B.H. Savitzky, et al., Ultramicroscopy 191 (2018), p. 56. 